\title{
The Automated Psychophysical Test (APT) for assessing age-diminished capabilities
}

\author{
A. SCOTT MCKNIGHT and A. JAMES MCKNIGHT \\ National Public Services Research Institute, Landover, Maryland
}

\begin{abstract}
In this report we describe a computer-based psychophysical test that can assess capabilities that have been shown through research to be related to both age and automobile accidents. The test operates with a standard personal computer. To facilitate its use with an elderly population, instructions are given aurally by means of a sound card as the exercises are demonstrated. Responses are registered with a joystick; the direction of the joystick's motion corresponds to the direction or location of stimuli on the screen to obviate the learning of response codes. The system is currently being used to study the diminished capabilities of elderly drivers who have been referred to a state licensing agency because of deficient driving behavior.
\end{abstract}

In this report we describe a set of measures that has been designed by the National Public Services Research Institute to assess perceptual and cognitive abilities that are involved in the safe operation of automobiles. The program was developed as a screening tool that could be used to identify subjects with psychophysical deficits. All of the abilities measured by the test are required in the operation of automobiles, and most have shown a relationship to automobile accidents. The program is designed to measure sensory abilities, including static, dynamic, high- and low-contrast visual acuity, and visual field; attentional abilities, including selective attention and attention sharing; perceptual abilities, including perceptual speed, visual tracking, field dependence, and motion detection; cognitive abilities, including information processing and short-term memory; and motor abilities, including simple and choice reaction time. The measures are divided into two categories: abstract measures, which are measures employing abstract symbols as stimuli, and driving measures, which are measures employing stimuli drawn from the highway traffic environment. In some cases the driving measures consist of videotaped footage of traffic scenes from the driver's point of view. Other driving measures are more abstract in nature, but use as stimuli symbols that are normally associated with driving.

The abstract measures are used for two reasons. First, research measures for studying the various sensory, attentional, perceptual, cognitive, and motor functions generally employ abstract symbols because of their simplicity and their freedom from the influences that meaningful symbols would invoke. Second, abstract symbols can be created and manipulated in such a way as to assess individual psychophysical functions, whereas responses to driving stimuli almost invariably involve a mixture of many different functions. Driving-related stimuli, on the

Address correspondence to S. McKnight, National Public Services Research Institute, 8201 Corporate Drive, Suite 220, Landover, MD 20785. other hand, have the advantage of face validity. Although one might not be able to identify the specific psychophysical shortcomings that contributed to a deficient driving response, there would be security in knowing that the deficient response was indeed one that was required in driving. Moreover, in any practical application in which a test is used in a way that affects drivers, face validity would be likely to give the measures greater credibility than would one that employs a set of abstract symbols. Finally, through correlations between the abstract and driving measures, it may be possible to associate the deficiencies in specific driving measures with their corresponding psychophysical deficits.

All instructions are presented in audio form; an audio card installed in the computer plays back prerecorded instructions. Along with the audio instructions are visual illustrations showing subjects what they will see and how to use a joystick to register their reactions. This system of instruction was chosen in an effort to reduce the amount of time necessary to learn how to perform each measure, thereby reducing the amount of time needed to gather meaningful data.

\section{ABSTRACT MEASURES}

The battery of abstract measures is arranged so that measures using the same targets and/or requiring the same reactions from the subjects are juxtaposed. This was done to streamline the instructions and reduce confusion for the subject. For each exercise the computer records response times and whether responses are correct, as well as other pertinent information. The subjects are instructed to respond to all stimuli as quickly as possible. The following is a description of the abstract measures by ability type.

\section{Measures of Sensory Abilities}

Static acuity. One letter, E, oriented in one of four positions (facing up, down, left, or right), appears and re- 
mains on the screen until the subject responds by pushing the joystick in the direction corresponding to the opening in the E. Presentation starts with Es that are $20 \times 20$ pixels and then proceeds to smaller sizes of $15 \times 15,10 \times 10$, and $5 \times 5$ pixels. Whenever the target is correctly identified the first three times it occurs at any level, the series proceeds immediately to the next smallest size. If the subject responds incorrectly to 5 trials at a given size, the program will proceed to the next test rather than to the next smallest size. If the preceding conditions are not met, all 10 targets are presented before going on to the next size. When any of these criteria (first 3 correct, 5 incorrect, or all 10 trials given) have been met for the last exercise (smallest target), the series proceeds to the next measure.

Dynamic acuity. This is essentially the same as the static acuity measure, except that the letter E moves left to right across the screen.

Low-contrast acuity. This is essentially the same as the static acuity measure, except that each target is a light gray $\mathrm{E}$ against a darker gray background.

Parafoveal target detection. A small white square appears in 1 of 10 locations around the screen. The square is displayed for $3 \mathrm{sec}$, or until the subject responds by pushing a red button on top of the joystick. The location of the square is varied randomly from one trial to the next.

Parafoveal form detection. An arrow appears in 1 of 10 positions around the screen and points in any of four directions (left, right, up, or down). The subject must deflect the joystick in the direction indicated by the arrow.

\section{Measures of Attentional Abilities}

Selective attention. One arrow appears; it is oriented in one of four directions (up, down, left, or right), and appears in one of four locations (top, bottom, left, or right).

In the first 10 trials, the subject is instructed to deflect the joystick in the direction indicated by the location of the arrow on the screen, regardless of the orientation of the arrow. In the second 10 trials, the subject is instructed to deflect the joystick in the direction that the arrow is oriented, regardless of its location on the screen. Finally, there are 10 more trials in which the subject must deflect the joystick in the direction of the arrow's location. This measure follows directly after the parafoveal form-detection measure, thus creating a series of tasks in which the subject must first respond to the orientation of the arrow (disregarding its location), then to its location (disregarding its orientation), then to its orientation, and then once again to its location.

Attention sharing. This measure is a combination of the visual tracking exercise (described below) and the parafoveal form-detection exercise (described above). A white cross appears and moves across the screen toward a white square; while the cross is approaching the square, an arrow appears. The subject must indicate the direction of the arrow with a deflection of the joystick in that direction. Simultaneously, the subject must evaluate the rate of closure of the cross toward the square, predict when they will intersect, and push the button on top of the joystick when the cross is completely within the square.

\section{Measures of Perceptual Abilities}

Perceptual speed. A matrix of large, high-contrast Es appears-five Es wide and four Es high. All the Es are oriented vertically (opening either up or down) except one, which is oriented horizontally (opening either left or right). The subject must locate the one $E$ that is horizontally oriented and push the joystick in the direction of that E's opening.

Visual tracking. A small square appears in each of five positions around the perimeter of the screen: center, top, bottom, right, and left. Simultaneously, a small cross, which is the same height and width as the square, appears above, below, to the right, or to the left of the square, and begins moving toward the square. The subject must stop the cross by pushing the button on top of the joystick when it reaches the square. The subject is instructed to stop the cross as close as possible to the point at which the cross is entirely within the square. The computer records the distance, in computer screen pixels, between the position at which the subject stops the cross and the true point at which the cross is entirely within the square.

Motion detection. A small white square appears at the left, right, top, bottom, or center of the screen and immediately begins moving up, down, left, or right. The subject must deflect the joystick in the direction that the square moved.

\section{Measures of Cognitive Abilities}

Information processing/missing $\mathbf{E}$. A matrix of large, high-contrast Es appears-five Es wide and four Es high. All the Es are oriented in one of three of the four possible orientations (up, down, left, or right). One of the four possible orientations is not represented. The subject is instructed to push the joystick in the direction corresponding to the one orientation that is not represented.

Information processing/digit matching. An eight-digit (target) number appears in the center of the screen. This number is surrounded by four more eight-digit (probe) numbers that are arranged above, below, to the right, and to the left of the central number. One of the probes contains the same digits that are in the target number, but in a different order. The subject is instructed to push the joystick in the direction of this probe.

Digit-span short-term memory. A string of four digits is presented, followed by a screen message that inquires whether a specific "probe" digit was in the string. Three such probe digits are presented. Next, a string of five digits is presented, followed by three probe digits. This continues with digit strings that are six, seven, and eight digits in length, each followed by three probe digits. The subject must deflect the joystick in the direction cor- 
responding to "yes" and "no," as indicated at the bottom of the screen, in order to indicate whether the probe digit was in the string.

\section{Measures of Motor Abilities}

Simple reaction time. A small white square appears in the center of the screen. The subject must push a button on top of the joystick as soon as possible after the square appears. On this and all other reaction time measures, the period of time between appearances of the square is varied randomly to prevent anticipatory responses.

Choice reaction time. One of four arrows, pointing up, down, right, or left, appears in the middle of the screen. The subject must deflect the joystick in the direction that the arrow points.

\section{DRIVING-RELATED MEASURES}

The following is a description of driving-related measures of perceptual/cognitive abilities. It should be mentioned at the outset that none of these measures can be as pure of an indication of ability as the abstract measure of the same ability. All of the following measures involve, to some extent, abilities other than the one under which it is described. Driving style will also play a great part in how subjects react. These measures, however, resemble natural driving much more closely, and therefore should not require the level of instruction that the abstract measures do. Measures in which subjects' driving ability is measured by an activity that closely resembles driving will also, obviously, have the advantage of face validity as compared with more abstract measures. Perhaps most importantly, a subject who does poorly, due to some diminished ability other than the one that a particular measure is intended to reflect, is still a hazard, and it is important to identify that driver no matter what the source of the problem. Before the driving-related measures begin, the subject is instructed in how to use the joystick to drive through the scenes and react as if he/she were actually driving a vehicle. The subject is also shown an illustration of a fictitious "Route 22" sign that has an arrow below it indicating a right turn. The subject is told that any time this sign is seen along the videotaped route, he/she should turn right at the next intersection to get to Route 22. In all the scenes, the subject sees a driver's point of view of the road ahead. Superimposed across the top of the screen is a mirror-image view of the road behind, which functions as a rearview mirror. For videobased measures, the program records joystick input information from which response times and appropriateness of response can be determined later. The following is a description of driving-related measures by ability type.

\section{Sensory Abilities}

Static acuity. The vehicle approaches a sawhorse that has been placed across its lane. As the vehicle comes closer to the sawhorse, the sawhorse gets bigger, appear- ing to emerge from the background. The point at which the subject detects the presence of the sawhorse is a function of the subject's ability to see the lines formed by the sawhorse against the ground.

Parafoveal target detection. A vehicle emerges from the subject's blind spot and cuts across the subject's path, forcing a reaction of either swerving or braking. The subject's reaction time is a function of the ability to detect an object that appears, in an unpredictable manner, in the subject's field of view.

Parafoveal form detection. This measure is based on computer graphics rather than video images. One of three shapes appears at random locations around the screen (much like the abstract form-detection measure)-a car, a boat, or an airplane. The subject is instructed to press the joystick button when, and only when, the car appears. As with the abstract measure, reaction time will be influenced by the ability to identify the form while it is still in the subject's parafoveal vision. Although this measure is only slightly more "driving related" than its abstract counterpart, the use of the car as a stimulus does provide an element of face validity that is missing in the abstract measure.

\section{Attentional Abilities}

Selective attention. There are several scenes that are designed to measure selective attention, and each involves driving on a section of roadway that includes some sort of "unimportant distraction" along either side. These distractions include a roadside air museum with vintage planes that are visible to the subject, a vintage car approaching in an oncoming lane, and a bicycle parked well off the road, with a pedestrian examining its chain.

Within each scene there is also a hazard or other type of stimulus that would require some sort of reaction (e.g., a "Route 22'" sign that the subject must follow). There is never more than one hazard or change of direction at the same time; however, the distractions, hazards, and direction changes could all appear at the same time. Latency in reacting to hazards and directions in the presence of distractions would indicate an inability to attend selectively.

Attention sharing. The attention-sharing measures are similar to the selective attention measure, except that there are no unimportant (e.g., purely distracting) stimuli. Hazards compete for attention with other hazards and directions, each requiring the possibility of a response. The inability to perceive and react to competing hazards or directions would indicate an inability to share attention. For example, in one case a "Route 22"' sign appears, requiring a right turn at the next intersection, and at the same time a pedestrian threatens to run into the vehicle's path. A driver who cannot share attention may be too intent on the pedestrian to notice the direction change, and therefore might miss the turn. In another case, a car begins to pass the vehicle along the left side, and at the same time a car starts to pull out in front. A driver who cannot share attention between cars ahead and cars in the rear- 
view mirror may swerve to the left and strike the car that is in the blind spot.

\section{Perceptual Abilities}

Perceptual speed. There are a number of perceptual speed measures; each involves a scene in which a hazard appears in such a way that its hazardous nature becomes more apparent as the scene develops. For example, in one case, the subject's vehicle is in the left of two lanes. Ahead in the right lane is a vehicle that signals and begins slowing for a right turn. Behind this vehicle, and just ahead and to the right of the subject's vehicle, is another car that will eventually pull around the first one in order to pass it. The speed with which the subject reacts to the second car as it cuts into the path ahead is, in part, a function of the speed with which a driver can perceive the chain reaction of events that will cause that driver to pass.

Visual tracking. The subject approaches an uncontrolled intersection at the same time as a car approaching from the right. In some cases the car is obviously slowing to stop. In other cases the slowing is not as obvious, and there is some doubt as to whether the car will stop. In some cases the car will not stop, and in other cases the car will cross so far ahead that it would be inappropriate to stop or slow down. The subject who does not react appropriately to the approaching car would be considered to be lacking in visual tracking ability.

Field dependence. This exercise uses six target screens and six probe screens. The target screens consist of one of three shapes representing the outlines of common traffic-sign shapes, in black on a white background. The shapes are an inverted triangle (yield sign), a circle with a line through it (international "no"), and a diamond (warning sign). For each target screen showing the simple shape, there is a probe screen that follows, which contains a complex multicolored pattern in which the target shape has been embedded.

The subject is instructed to push the joystick toward the corner of the probe screen in which the target shape has been embedded. The joystick button can be pressed to review the target screen once the probe screen is being displayed. Each target screen is displayed and then followed by a probe screen. The probe screen is displayed for $15 \mathrm{sec}$ or until the subject responds. The subject performs two trials for each of the three target types.

Motion detection/angular. While driving down a residential street, a white car against a dark background rolls out of a driveway into the path of the subject's vehicle. The driveway is on a hill, so the speed of the car increases as the scene progresses. The speed with which the subject reacts is a function of (1) the ability to detect motion outside the center of vision, and (2) the speed that the moving object must attain before being detected as moving by the subject.

Motion detection/depth. A vehicle that is ahead of the subject's vehicle begins to slow down so that the subject overtakes it. The vehicle ahead coasts in a low gear so that it slows fairly quickly, yet no brake lights come on to cue the subject that it is decelerating. The reaction time is a function of the subject's ability to detect the motion of the vehicle ahead in relation to his/her own vehicle.

\section{Cognitive Abilities}

Information processing/icon matching. This measure is based on computer graphic images, and derives its relationship to driving from the use of traffic signs as stimuli. There are five categories of signs: regulatory signs (Stop, No U-Turn, Do Not Enter, No Right Turn, Yield); lane-use control signs (Keep Right, Left Turn Only, Right Turn Only, Left Only, Straight Only, Left, Right, or Straight); general service signs (Handicapped Parking, Roadside Picnic Area, Airport, Hospital, Food); pictographic warning signs (Deer Crossing, Farm Machinery Crossing, Bicycle Crossing, Pedestrian Crossing, Slippery When Wet); and road-shape signs (Winding Road, Side Road, Y-Intersection, Cross Road, Right Turn).

For each type of sign, the subject sees a screen in which there is a (target) cluster of three signs in the center of the screen and four more (probe) clusters of three signs each-one in each corner of the screen. One of the probe clusters matches the target cluster exactly. The subject must indicate, with the joystick, which corner contains the cluster that matches the cluster in the center.

Icon short-term memory. This is similar to the icon information-processing measure, except that the subject does not see the target and probe stimuli at the same time. For each type of sign, the subject first sees a (target) screen displaying a cluster of three signs, followed by a (probe) screen displaying four clusters-each in a different corner of the screen. The subject must indicate, with the joystick, which corner contains the cluster that matches the first cluster they saw. After the subject sees, and responds to, target/probe combinations for each type of sign, five more probes appear (without new targets), one for each type of sign. These probes are not necessarily the same as the first probes that were shown. For this second round of probes, the subject must indicate the corner containing the target cluster that was seen earlier. This provides a measure of short-term memory with backward masking.

\section{Motor Abilities}

Simple reaction time. In a video-based simple reaction time measure, a subject follows behind a car that puts on its brakes. The simple reaction time is the time that it takes for the applicant to react by pulling back on the joystick.

A second simple reaction time measure is based on computer graphics, in which the subject sees a roadway from the driver's point of view. The subject's vehicle is on a two-lane road, with a solid yellow line separating the subject's lane from the oncoming lane. A car is just ahead of the subject's vehicle. After a short time, the brake lights of the vehicle ahead come on, and the subject must brake as quickly as possible by pulling back on the joystick.

Choice reaction time. This measure also uses programmed computer graphics rather than actual in-traffic video footage. The subject's vehicle is in the center of 
three lanes. Ahead are three identical cars, one in each lane. After a short time, the brake lights on some of the vehicles come on. The subject is instructed to use the joystick to swerve left if the center and right cars brake, swerve right if the center and left cars brake, and stop if all three cars brake.

\section{EQUIPMENT NEEDED}

The abstract measures and computer-based, drivingrelated measures are programmed for use on an MS-DOScompatible computer with an 80386 or better processor, running at $16 \mathrm{MHz}$ or faster. The computer monitor must be capable of displaying color images of VGA resolution or higher. The use of audio instructions requires the use of an audio card. The system is currently programmed to use one of two audio cards from Video Associates Labs of Austin, TX.

The video-based, driving-related measures are currently programmed for use with a Pioneer Model LDV 4200 videodisk player, a videodisk containing the driver'spoint-of-view video scenes, and a Microkey video overlay card, also from Video Associates Labs. The video overlay card is used so that the videodisk-based video can be played on the computer monitor. An alternate version of the program substitutes an NEC PC-VCR (a computercontrolled Super-VHS videotape deck) for the videodisk player. This program also eliminates the need for the video overlay card by sending the videotape-based video to a second (video, rather than computer) monitor.

\section{AVAILABILITY}

The APT program is available from NPSRI for the cost of disks, plus shipping and handling. Contact S. McKnight at National Public Services Research Institute, 8201 Corporate Drive, Suite 220, Landover, MD 20785; phone, 301-731-9891; fax, 301-731-6649; e-mail, 71341,261@ compuserve.com.

\section{CONCLUSIONS}

We believe that the APT program is a valuable tool for the measurement of psychophysical abilities. Although it was developed to be a screening tool, an effort has been made to make it flexible enough to be used by researchers who want to measure any of the psychophysical abilities described in this paper. It is possible to administer a subset of the entire battery. Relevant data, including response times and correctness of response, are recorded to a computer file on a trial-by-trial basis. This gives users the maximum flexibility in using the data in subsequent analysis.

As of this writing, no large-scale validation of this measure has been made, although there are plans to do so in the near future. 